ROCZNIKI TEOLOGICZNE

Tom LXVIII, zeszyt 3 - 2021

DOI: https://doi.org/10.18290/rt.21683-3

\title{
DIE PERSONALISTISCHE GRUNDLAGE DER KIRCHLICHEN LEHRVERKÜNDIGUNG BEZÜGLICH DER PERSONEN MIT LEBENSVERKÜRZENDEN ERKRANKUNGEN
}

\author{
THE PERSONALISTIC BASIS OF THE TEACHING OF THE MAGISTERIUM REGARDING \\ PERSONS WITH PATHOLOGIES "INCOMPATIBLE WITH LIFE"
}

\begin{abstract}
A b s t r a c t. The wave of protests following the decision of the Polish Constitutional Court of October 22, 2020 about the unconstitutionality of the embryopathic indication for abortion can be seen as a challenge for the Catholic Church to clarify its pro-life doctrine with regard to the protection of the weakest and to raise it anew. A support for this is the letter of the Congregation for the Doctrine of the Faith Samaritanus bonus of July 14, 2020. The present article takes up the said doctrinal teaching in order to synthesize and justify the moral view represented in this teaching relating to abortion, especially in the case of pathologies "incompatible with life". The guiding principle is the personalistic view of human being, according to which the person should be at the center of all ethical decisions. From the consideration of the individual aspects of the question raised, certain ethical imperatives follow, which are considered in the conclusions.
\end{abstract}

Key words: "incompatible with life"; abortion; person; bioethics; relationality.

\section{EINLEITUNG}

Das Urteil des polnischen Verfassungsgerichts vom 22.10.2020 über die Verfassungswidrigkeit der bisher in der Gesetzgebung des Landes geltenden Ausnahme der Straffreiheit einer Abtreibung im Falle der hohen Wahrschein-

Dr. theol. ANDRZEJ KUCIŃSKI - in Moraltheologie an der Universität Bonn promoviert, Priester des Erzbistums Köln, Mitarbeiter der Glaubenskongregation, wohnhaft in Rom; e-mail: a.kucinski@live.com; ORCID: https//orcid.org/0000-0001-5327-1670.

Ks. dr ANDRZEJ KUCIŃSKI - doktor teologii moralnej na Uniwersytecie w Bonn, ksiądz archidiecezji kolońskiej, pracownik Kongregacji Nauki Wiary, mieszka w Rzymie; e-mail: a.kucinski@live.com; ORCID: https//orcid.org/0000-0001-5327-1670. 
lichkeit einer schweren und irreversiblen Beeinträchtigung des Fötus oder einer lebensverkürzenden Krankheit sowie die heftige Protestwelle gegen diesen Gerichtsspruch, wie sie unmittelbar danach entflammte, bilden einen herausfordernden Anlass, um sich mit dieser Problematik moraltheologisch vertieft zu beschäftigen. Das Thema der lebensverkürzenden Krankheiten wurde gerade in einem im September 2020 erschienen und auf den 14. Juli 2020 datierten Dokument der Glaubenskongregation Samaritanus bonus , über die Sorge an Personen in kritischen Phasen und in der Endphase des Lebens" als Teilfrage behandelt. Die Analyse der einschlägigen lehramtlichen Verkündigung ergibt, dass das Verbot der direkt gewollten Tötung von Menschen mit lebensverkürzenden Krankheiten den Tatbestand einer absolut verwerflichen, durch nichts zu rechtfertigenden Vernichtung eines Ungeborenen erfüllt. Als Grundtenor und anthropologische Grundlage der bisherigen Lehrverkündigung kann die personalistische Sicht des Menschen seitens der katholischen Kirche identifiziert werden.

In dem vorliegenden Artikel handelt es sich nun um eine moraltheologische Synthese der kirchlichen Lehre bzgl. der genannten Problematik im Rahmen dieses personalistischen Ansatzes, um in einem zweiten Schritt eine entsprechende Begründung der kirchlichen Position zu versuchen und sie abschließend als plausible Option des Herangehens an ethische Herausforderungen darzustellen.

\section{DIE ALLES ENTSCHEIDENDE TELEOLOGIE}

Es ist der Einschätzung von Elio Sgreccia zuzustimmen, gemäß dem die „,postmetaphysische“ Bioethik sich in eine Sackgasse des kontinuierlichen Urteilswandels begebe, wenn sie bei Behandlung von ethischen Fragen von anthropologischen und letztlich von ontologischen Problemen absehe ${ }^{1}$. Das Gegenteil ist aber der Anspruch der katholischen Kirche, die sich dabei einerseits dem natürlichen Sittengesetz und andererseits der göttlichen Offenbarung verpflichtet weiß. Im Zentrum dieses Ansatzes steht die Achtung vor jedem menschlichen Lebe-

\footnotetext{
1 „It seems that postmetaphysical bioethics has perceived that so-called bioethical problems are often more accurately anthropological problems, but it does not consider it feasible to take an ontological approach to anthropology. It prefers an ethical route - meaningful, but insufficient for preventing science from being considered the only form of knowledge and human nature from being subjected to a continual process of redefinition“. - Elio Sgreccia, Personalist Bioethics. Foundations and Applications (Philadelphia PA: The National Catholic Bioethics Center, 2012), 371.
} 
wesen als Person, der vom ersten Augenblick ihrer Existenz an unveräußerliche Rechte natürlich und nicht mittels Zuschreibung durch Dritte zukommen, allem voran das Lebensrecht, das Fundament aller anderen Rechte. Die Menschen- bzw. Personenwürde, die hier deckungsgleich sind, verbietet jeden verzweckenden Umgang mit dem Menschen, sofern er auf irgendeinen Zweck, der nicht er selbst ist, reduziert wird. Dies erfordert jedoch eine schwerwiegende Voraussetzung gerade zu Beginn des Lebens, um die Freiheit jeder Person von Anfang an zu gewährleisten: „only a teleological view of reality based on creation can protect causal origin, which in this case is not seen as blind chance but as Providence. Dependence on an equal can enslave us, whereas dependence on God - due to the absolute distance between Creator and creature - is a source of freedom"2. Teleologisch auf den Menschen zu schauen bedeutet somit, seine eigene, intrinsische Zielgerichtetheit zu respektieren, die nicht durch Menschen festgelegt wurde und deshalb auch nicht durch Menschen manipuliert werden darf. Die Zielgerichtetheit des Menschen zu kennen setzt wiederum grundlegende anthropologische Überlegungen voraus, welche man nicht den pragmatischen Lösungen opfern sollte, will man zu verantwortbaren und dauerhaften Ergebnissen kommen. Von daher kann man die lehramtliche Beteuerung verstehen, ausnahmslos jedem menschlichen Lebewesen die unbedingte Achtung entgegenzubringen, um von hier aus die konkreten Fragestellungen anzugehen.

Vor diesem Hintergrund und in Bezug auf das moralische Problem der Menschen mit lebensverkürzenden Erkrankungen muss man grundsätzlich unter dem Oberbegriff der Abtreibungsfrage - drei verschiedene Themenbereiche in Betracht ziehen: Pränataldiagnostik, ,therapeutische“ Abtreibung und „eugenische“ Abtreibung.

\section{MORALISCHE ZULÄSSIGKEIT DER PRÄNATALDIAGNOSTIK}

Im Fall der Pränataldiagnostik kann man wiederum drei moralisch relevante Elemente unterscheiden: die Handlung an sich, die Absicht des Haupthandelnden und die Verantwortung des Mitwirkenden (Arztes bzw. des Begleitpersonals). Evangelium vitae, Nr. 63, stellt eine doppelte Bedingung für die Unbedenklichkeit der entsprechenden diagnostischen Verfahren fest: der Ausschluss von „unverhältnismäßigen Gefahren“ für Mutter und Kind und die lautere Absicht, durch solche Verfahren eine frühere und deshalb vielleicht

\footnotetext{
2 Ebd., 370.
} 
erfolgversprechende Therapie zugunsten des Kindes vorzunehmen. Im Zentrum steht also das eigene Telos des Kindes, das freilich mit dem Wohl der Mutter gemeinsam gedacht wird. Wird aber die Absicht zum Ausdruck einer eugenischen Mentalität und das diagnostische Verfahren zum Ausgangspunkt für eine mögliche Selektionsabtreibung, wird die Qualität der Handlung „verdorben“, entsprechend dem Grundsatz: bonum ex integra causa, malum ex quocumque defectu. Schließlich bleibt die Frage der Verantwortung des/der Mitwirkenden, der/die die betroffene Mutter über die Ergebnisse der Diagnostik informieren muss. Sgreccia verweist in dem Zusammenhang auf verschiedene Formen der cooperatio ad malum und schließt, dass der/die Leiter/-in des diagnostischen Verfahrens lediglich indirekt materiell mitwirke (was moralisch unbedenklichen Charakter hat), indem er die eventuelle böse $\mathrm{Ab}$ sicht des/der Hauptakteur/-in nicht teile und es zwischen der diagnostischen Handlung und der möglichen, bösen Abtreibungshandlung eine eindeutige Trennung gebe ${ }^{3}$. Gleichzeitig ist jedoch klar, dass sich das medizinische Personal nicht in einer ethisch neutralen Situation befindet und bei allem Respekt vor der Freiheit und Verantwortung des Anderen von seiner eigenen Freiheit und Verantwortung für das Leben, das hier möglicherweise aufs Spiel gesetzt wird, in Anspruch genommen wird ${ }^{4}$.

\section{ABTREIBUNGSFRAGE IM FALLE DER GEFÄHRDUNG FÜR GESUNDHEIT ODER LEBEN DER MUTTER}

Im zweiten Fall handelt es sich um ein Argument für die Abtreibung bei embryopathischer Indikation und erst recht im speziellen Fall der lebensverkürzenden Erkrankung: die Gefährdung der Mutter. In der polnischen Gesetzgebung sind diese zwei Indikationen jedoch getrennt, weil zwischen den beiden kein zwingender Zusammenhang bestehen muss. Zudem ist der im Fall einer realen Gefährdung der Mutter benutzte Begriff der ,therapeutischen“ Abtreibung irreführend: „First of all it must be said that the adjective therapeutic is inaccurate: in fact, it is not a matter of therapy except in a broad and improper sense. [...] it is not a matter of treating an active illness in the case in question, but rather of killing the fetus to avoid aggrava-

\footnotetext{
${ }^{3}$ Vgl. ebd., 362.

${ }^{4}$ Vgl. ebd., 363-4.
} 
ting the mother's health or risking her life" 5 . In diesem Fall lässt sich jedoch ein breites Spektrum von moralischen Entscheidungssituationen ausmachen, je nachdem, wie das Verhältnis der Schwangerschaftsfortsetzung zur Gesundheit der Mutter eingeschätzt wird, wie sicher das Risiko ihres Todes ist, ob ihr Tod automatisch den Tod des Kindes bedeuten muss, wie hoch der Schaden für die Gesundheit der Mutter sein soll (wenn es sich nicht um die Todesgefahr handelt), was man unter Gesundheitsgefährdung überhaupt versteht und ob man die psychologischen Auswirkungen der Geburt eines schwerkranken Kindes bei der Mutter nicht bereits als deren Gefährdung ansieht, also die „eugenische“ Abtreibung zu einer „therapeutischen“ macht ${ }^{6}$.

Zur moralischen Beurteilung dieser Art der Abtreibung, der Beurteilung, die vielleicht am schwierigsten zu vermitteln ist, gehört als Grundlage die Anerkenntnis, dass die menschliche Person den höchsten Wert in der Welt ausmacht und jedes zeitliche Gut bzw. jede ökonomische Betrachtung transzendiert $^{7}$. Es bedeutet auch, dass die Person der Gesellschaft vorausgeht, weil die Gesellschaft aus und durch Personen gebildet wird. Folglich kann man nicht den Wert der Gesellschaft als ganzer und den Wert der Einzelperson als solcher gegeneinander abwägen, da jede Person innkommensurabel und ihre Würde nicht quantifizierbar ist. Das Leben ist aber die unabdingbare und primäre Grundlage aller anderen personalen Werte, was bedeutet, dass man über diese Werte nicht sprechen kann, solange man den grundlegenden Wert des Lebens der Person abspricht. Nur vor diesem Hintergrund lässt sich anthropologisch die ,therapeutische“ Abtreibung wirksam hinterfragen, abgesehen davon, dass in konkreten Fällen die Entscheidung zuungunsten des Kindes oft zu schnell fällt, ohne dass reale Alternativen überprüft werden ${ }^{8}$. In jedem Fall wird dann das Kind instrumental-funktionalistisch in Bezug auf Gesundheit und Leben der Mutter gesehen. Außerdem wird dabei ausgeblendet, dass die Mutterschaft - wie jede andere anthropologische Aufgabe im Leben - natürlicherweise Risiken für die eigene Gesundheit oder sogar Leben mit sich bringen kann. Die sich als human verstehende Wissenschaft sollte

\footnotetext{
5 Ebd., 447.

${ }^{6}$ Ebd., 448. Vgl. auch Adriano Bompiani, „Indicazioni all'aborto «terapeutico»: stato attuale del problema“, in Aborto. Riflessioni di studiosi cattolici, hrsg. v. Angelo Fiori, Elio Sgreccia (Milano: Vita e Pensiero, 1975), 191-218.

${ }^{7}$ Bei den folgenden Grundsatzüberlegungen in diesem Absatz wird im Wesentlichen auf Sgreccia, Personalist Bioethics, 450-2 verwiesen.

8 „In light of advances in science and medical care, many of these [health] 'indications' have lost their compelling force“. - ebd., 450.
} 
demnach dem Wohl der Person(en) dienen und zur Vermeidung von derartigen Risikosituation nur mit Mitteln beitragen, die recht sind, d. h. dieses Wohl (und zwar das Wohl aller beteiligten Personen) als Maßstab nehmen.

Auf dieser Grundlage ist in der konkreten Situation zuerst notwendig, alles daran zu setzen, sowohl Mutter als auch Kind zu retten. Insbesondere darf man keinesfalls Ausnahmen für irgendeine direkte Vernichtungsaktion des menschlichen Lebens einführen, will man nicht in einer willkürlichen Praxis landen (denn Ausnahmen lassen sich grundsätzlich immer weiter ausdehnen). Ist der Tod der Mutter unvermeidlich, aber die Hoffnung auf Rettung des Kindes begründet, kann man versuchen - z. B. mittels künstlicher Aufrechterhaltung von Lebensfunktionen der sterbenden Frau -, das Kind zum Stadium zu bringen, in dem sein Leben außerhalb der Gebärmutter möglich wird ${ }^{9}$. In dem umgekehrten Fall, wenn die Schwangerschaftsfortsetzung voraussichtlich weder Mutter noch Kind rettet, ihre Unterbrechung aber zumindest das Leben der Mutter retten könnte, gilt - unter der Voraussetzung einer personalistischen Anthropologie - dennoch nach wie vor, dass eine direkte Abtreibungsaktion unterbleiben muss. Jegliche Aktion zur Rettung der Mutter darf den Tod des Kindes höchstens als unerwünschten Nebeneffekt zulassen, den man zwar voraussehen kann, aber ihn weder als Ziel noch als Mittel anstrebt. Dann aber kann man nicht von der „Abtreibung“ sprechen: „It is not an abortive action aimed directly at the fetus but rather an action necessary to preserve the life of the mother which is aimed at some part of her body “10.

\section{DIE VERWERFLICHKEIT DER „EUGENISCHEN“ ABTREIBUNG}

Im dritten hier zu behandelnden Fall, dem eigentlichen Kontext der lebensverkürzenden Erkrankungen, handelt es sich um die sog. ,eugenische“ Abtreibung. Derzeit wird diese zwar nicht mehr als solche bezeichnet, um Konnotationen zur NS-Euthanasiepraxis, die das Ziel verfolgte, die „Rassenreinheit“ zu bewirken und die Gesellschaft von den Lasten zu „befreien“, die mit der Sorge für körperlich und geistig eingeschränkte Personen verbunden sind, zu vermeiden ${ }^{11}$. Dennoch handelt es sich der Sache nach weiterhin um die Abtreibung von fehlgebildeten oder behinderten Individuen, eine Handlung, die

\footnotetext{
${ }^{9}$ Vgl. ebd., 454.

${ }^{10}$ Vgl. ebd., 470-1.

11 Ebd., 455.
} 
oft als Befreiung ihrer selbst von einem Leben mit geringer "Qualität“ dargestellt wird, im Grunde aber doch die Absicht, die Gesellschaft und die Familien der Betroffenen zu entlasten, vermuten lässt. Es handelt sich also um ein auf jeden Fall utilitaristisches Argument, welches das Leben einer Person gegen einige mit diesem Leben inkommensurable Werte abwägt. Aus personalistischer Sicht steht aber fest: ,the presence of a deformity or a handicap in no way diminishes the ontological reality of the unborn child; indeed, the presence of an impairment - such as a disease - in a human subject calls for protection and assistance all the more, in the name of solidarity" ${ }^{\text {"12. Generell }}$ sollte daher darauf hingearbeitet werden, dass solche Fehlbildungen und schwere Erkrankungen bereits im pränatalen Stadium behandelt werden können und dass die Familien entsprechende, institutionelle Hilfen von der Gesellschaft erhalten, um die Lasten zu lindern, die das Leben mit und die Sorge an solchen Personen (wenn sie denn nach der Geburt noch leben) mit sich bringen. Die tatsächliche Erfüllung dieser ethischen Imperative beeinflusst jedoch nicht - aus denselben anthropologischen Gründen, die oben dargestellt wurden - die ethische Unzulässigkeit der direkten Tötung von Föten, gemäß dem Argument, dass solche Menschen, vereinfacht formuliert, doch „,sowieso sterben würden“. Weder ökonomische Lasten für die Gesellschaft noch das Drohen einer emotionalen und wirtschaftlichen Beschädigung der Familie noch die verringerte physische Qualität des Lebens aller Betroffenen können die Vernichtung eines menschlichen Subjekts mit voller Menschenwürde und Recht auf Leben rechtfertigen. Dessen tatsächliche Fehlentwicklung steht auf einem anderen Niveau als sein ontologischer Status als Mensch und damit als Person. Sein bisweilen erschreckendes, von vielen als „unmenschlich“ empfundenes Aussehen kann nicht das wirklichkeitsgerechte Urteil korrigieren, dass der Personenstatus unabhängig von der Vollentwicklung aller personalen Qualitäten bleibt, an denen wir die Person erkennen. Denn: „The subject is biologically and ontologically defined in this reality and therefore constitutes the primary and indispensable foundation for the realization of all the other values and rights of the person“"13.

\footnotetext{
12 Ebd.

13 Ebd., 360.
} 


\section{SOZIALE DIMENSION DER TÖTUNG \\ VON UNGEBORENEN MIT LEBENSVERKÜRZENDEN KRANKHEITEN}

Bei der Betrachtung des ontologischen Status von Menschen unabhängig von ihrem Gesundheitszustand kommt überdies der Gedanke auf, dass die Abtreibung - gerade an Personen mit schweren Erkrankungen - ebenso die soziale Dimension umfasst, insofern sie relationalen Charakter trägt und öffentliche Wirksamkeit entwickelt. Nicht nur ist hier mit verschiedenen Folgen, auch Spätfolgen für die emotionale, psychische, moralische und gesundheitliche Stabilität der Frau, ihrer Familie und ihrer Umgebung zu rechnen. Es wird außerdem in die Gesellschaft hinein die Erkenntnis transportiert, dass das Recht der Schwachen durch die Macht der Starken unter bestimmten Bedingungen, „,bei Bedarf“, relativiert werden dürfe. Unterbleibt der rechtliche Schutz in solchen Fällen, hat die geltende Gesetzgebung eine negative formative Auswirkung auf das Gewissen der Bevölkerung; es verbreitet sich die Überzeugung: Was rechtlich zugelassen ist, kann auch moralisch nicht völlig verwerflich $\operatorname{sein}^{14}$. Insofern kann man das Problem der Abtreibung nicht auf das individuelle psychologische Problem der Frau reduzieren, stattdessen entwickelt diese eine relationale Dynamik, die weitere, unheilvolle Kreise zieht $^{15}$.

Der Status der moralischen Subjekte, die allen Menschen zukommt, zwingt jeden einzelnen, die anderen Subjekte in ihrer Andersheit ernst zu nehmen, auch wenn diese für ihre Interessen nicht eintreten können. Abtreiben heißt aber die Relationalität in Bezug auf solche Menschen zu verweigern, und damit auch einen Teil der eigenen menschlichen Natürlichkeit zu negieren, zu der auch zufällige und nicht nur freigewählte Relationalität notwendigerweise gehört. Indem ich den Anderen töte (töten lasse), beraube ich mich selbst des Rechtes, mit dem Anderen meiner selbst konfrontiert zu werden. Eine weitgehende Schlussfolgerung von D'Agostino gibt in diesem Zusammenhang Wesentliches zu bedenken: Die Abtreibung sei ,an archetypal emergence of that profound desire to deny relationality, in which the human

\footnotetext{
${ }^{14} \mathrm{Vgl}$. „The law must intervene in the matter of abortion because in general it has the duty to intervene every time the relational equilibrium is disturbed, every time that the concerns of the weak are threatened by the pretensions of the strong". - Francesco D' Agostino, Bioetica (Torino: Giappichelli, 1996), 277, zitiert nach: Sgreccia, Personalist Bioethics, 446.

15 Vgl. Francesco D’Agostino, Parole di bioetica (Torino: Giappichelli, 2004), 6; zitiert nach: Sgreccia, Personalist Bioethics, 447.
} 
intolerance of one's own creatureliness (and that of others) is manifested"16. Demzufolge kann das Argument, dass man die Frauen „Zwingen“ würde, sterbenskranke Kinder zur Welt zu bringen und dass dies „unmenschliches“ Handeln bedeute, falsifiziert werden: Wenn es etwas überhaupt gibt, das die Mutter zum Austragen eines kranken Kindes ,zwingt“, dann ist es ihre menschliche Natur selbst, in der der Umgang mit der Andersheit des Anderen zur Normalität gehört. Indem die Frau selbst Mensch und Person ist, kann und soll sie das Leben der anderen Menschen in dem Maße schützen, in welchem sie darauf Einfluss hat.

In Kontext der lebensverkürzenden Erkrankungen ist außerdem auf eine oft nicht bedachte Widersprüchlichkeit hinzuweisen ${ }^{17}$. Während einerseits die internationalen Dokumente die völlige Würde der geborenen Menschen mit Behinderungen wie selbstverständlich behaupten und die Gesellschaft immer aufmerksamer gegenüber ihren Bedürfnissen wird, inklusive verschiedener Formen von Unterstützung, wird andererseits mittels ,eugenischer“ Gesetzgebung massiv darauf hingearbeitet, dass solche Menschen überhaupt nicht geboren werden. Manche Mütter von Kindern mit Down-Syndrom müssen heute bereits die Erfahrung machen, dass ihnen argwöhnisch und unfreundlich begegnet und kolportiert wird, „man hätte das Problem ja lösen können“. Eine solche Mentalität erzeugt Druck auf die Schwächsten in der Gesellschaft und muss notwendigerweise in diverse Formen der Euthanasiebefürwortung münden. Die Eliminierung von schwerkranken Personen verunmöglicht jedoch auch die effektive Untersuchung von deren Krankheiten, die zum Ziel haben könnte, sie zukünftig frühzeitig und wirksamer zu behandeln.

Schließlich, abgesehen von allen anderen unheilbaren Erkrankungen, die aber nicht lebensverkürzend sind, wird im Falle der Föten, die in absehbarer Zeit sterben werden, argumentiert, dass man mit dem Schwangerschaftsabbruch nur den sicheren Tod antizipiert und somit qualitativ nichts hinzufügt, aber die Mutter von der Konfrontation mit dem fehlgebildeten Kind entlastet. Diese Überlegung verkennt jedoch die Belastung für Frau und ihre soziale Umgebung, die im Falle der Abtreibung empirisch feststellbar ist. Außerdem stellt sie sich nicht dem Faktum, wie eine Abtreibung eigentlich vonstattengeht und welches Leid sie ihrem schmerzempfindenden „Objekt“ zumutet. Nicht zuletzt missachtet sie den ethischen Unterschied zwischen

\footnotetext{
${ }^{16}$ D’Agostino, Bioetica, 275; zitiert nach: Sgreccia, Personalist Bioethics, 447. (Hervorhebung im Original).

${ }^{17}$ Vgl. Sgreccia, Personalist Bioethics, 360.
} 
einer direkten Tötung und der Begleitung im Sterben, welcher ja immanent einen qualitativen und nicht bloß quantitativen Charakter trägt. Denn einen Unschuldigen vorsätzlich zu töten heißt, die Personenwürde des Anderen zu verletzen, während demgegenüber eine schmerzlindernde Begleitung in seinem unvermeidlichen Sterbeprozess seine Selbstzwecklichkeit zu respektieren bedeutet.

\section{SYNTHETISCHE BEGRÜNDUNG DER KATHOLISCHEN ABLEHNUNG JEGLICHER ABTREIBUNGSPRAXIS}

Zusammenfassend ergeben sich unter der Voraussetzung einer anthropologischen Sicht, in der die Person als höchstes Gut im Zentrum steht, folgende Aspekte eines moraltheologischen Widerspruchs zur Abtreibung, insbesondere bei Subjekten mit geringer Lebenserwartung ${ }^{18}$ :

1) Es ist eine empirisch feststellbare Tatsache, dass das menschliche Leben mit der Zeugung beginnt. Der in der Verschmelzung von Eizelle und Spermium entstandene Organismus ist eine genetisch abgeschlossene und von seinen Eltern abgetrennte neue Lebenseinheit, genießt daher von Anfang an den Status der Person und damit eine unantastbare Personenwürde, unabhängig davon, dass einzelne Personalitätsmerkmale wie Rationalität und Selbstbewusstsein sich erst im Laufe der Zeit oder - wie im Falle von lebensverkürzenden Erkrankungen - vermutlich gar nicht entwickeln werden. Denn die Person transzendiert ihre eigenen Akte. Und nach der Zeugung sind aus biologischer, anthropologischer und philosophischer Sicht gar keine neuen „Qualitätssprünge“" zu erwarten: Es kann sich kein anderes Wesen aus dem entwickeln, was als Mensch gezeugt wurde. Die Person ist man immer schon im Akt oder gar nicht.

2) Jedes menschliche Lebewesen hat das gleiche Recht auf Leben, denn der Respekt vor dem biologischen Leben ist die Voraussetzung für allen anderen Respekt, den man der Person entgegenbringen kann. Man darf das eine Leben gegen das andere nicht abwägen, denn Kriterien wie Gesundheit, Lebensqualität, Fertigkeiten, Intelligenz usw. bedingen und relativieren die Menschenwürde nicht. Sobald biologisch feststellbar ist, dass man es mit einem lebendigen Menschenorganismus zu tun hat, gelten für ihn die gleichen Rechte wie für einen „vollständig entwickelten“ erwachsenen Vertreter der

\footnotetext{
18 Vgl. die Synthese von ebd., 471-4.
} 
Menschenart. Menschenwürde ist deckungsgleich mit Personenwürde. Das Leben der Mutter wiegt nicht mehr als das Leben ihres Kindes und umgekehrt.

3) Die staatliche Gesetzgebung kann nicht neutral bleiben, wenn es sich um Konfliktsituationen handelt, in denen von der Sache her die Rechte der Schwächeren den Interessen der Stärkeren geopfert werden sollen, unabhängig davon, welche positiven Intentionen dem Vorhaben zugeschrieben werden können.

4) Die Schwäche eines Embryos und eines Fötus - sein grundsätzliches Angewiesensein auf die Hilfe von außen, verschärft durch die zusätzliche Schwäche aufgrund der lebensverkürzenden Krankheit - ist ein Grund, um ihm mit besonderer Achtung zu begegnen und keine Begründung einer Tötung. Sie erlegt den „Starken“ zusätzliche Pflichten auf, denn jede Person ist durch ihr bloßes Dasein eine Verpflichtung für die anderen. Sich dieser Verpflichtung willentlich zu entziehen, hieße, aus der humanen Gemeinschaft aussteigen zu wollen und damit etwas Konstitutives an seiner eigenen Identität zu verlieren: die Relationalität mit dem Anderen seiner selbst. Solange der Mensch sich als Mensch versteht, ist er von Natur aus gehalten, die Andersheit des Anderen auszuhalten. In diesem Sinne ist jede Person eine Belastung für die Umgebung und die Gesellschaft. Der Grad dieser Belastung, der immer relativ bleibt, darf niemals über deren Lebenswürdigkeit entscheiden.

\section{SCHLUSSFOLGERUNGEN}

Die Vehemenz der ersten Proteste nach dem Urteil des polnischen Verfassungsgerichts vom 22.10.2020 über die konstitutionelle Nichtvereinbarkeit der embryopathischen Indikation zur Abtreibung mag viele der Katholiken, die der lehramtlichen Verkündigung weiterhin vertrauen, tief bekümmern. Sie bietet jedoch Anlass zum tieferen Überdenken dessen, was offensichtlich auch für immer weniger Gläubige als selbstverständlich gilt und nun noch mehr verloren zu werden droht. Es hat sich jedenfalls nicht die Lehre der Kirche geändert. Es ändern sich vielmehr die Gesellschaft und die Mentalität der Menschen, die immer öfters für oberflächliche, emotionsgeladene Argumentationen anfällig werden und den Zugang zu entsprechenden Begründungsmustern nicht finden. Glaubensbewusste Katholiken sollten sich dadurch herausgefordert sehen, um für die ihnen anvertraute Wahrheit vom Menschen einen solchen Zugang erneut zu schaffen. Dieser Zugang gilt auch und gerade für das so schwierige Thema wie die lebensverkürzenden Krankheiten. Das Doku- 
ment der Glaubenskongregation Samaritanus bonus bietet dafür entscheidende Stützen.

Hierzu gilt zunächst die richtige Nomenklatur zu gebrauchen. Es ist schon viel damit gewonnen, wenn man sich verbittet, im öffentlichen Diskurs in Bezug auf solche Menschen so abwertende Bezeichnungen wie „Zellklumpen“ oder gar „Monster“ zu verwenden. Die Sprache formt das Bewusstsein: Je mehr man in dem Zusammenhang ohne Abstriche auf Begriffe zur Bezeichnung dieses Lebens wie „Kinder“, „Menschen“ und „Personen“ insistiert, desto schwerer wird es, ihre Abtreibung als alleiniges Problem der Frau im Sinne des Abtreibungsbefürwortungsslogans „Mein Bauch gehört mir!“ durchgehen zu lassen. So kann auch das Argument der zu hohen Belastung für die Frau entkräftet werden. Denn es wird klar, dass sich hier von zwei unterschiedlich zu gewichtenden Werten handelt: die Selbstbestimmung der Frau steht nicht auf dem gleichen Niveau wie das Leben ihres Kindes. Insofern stellt Samaritanus bonus mit der Bezeichnung „kleine Patienten“ für solche Kinder in Bezug auf ihren ontologischen Status als Personen vieles noch einmal klar.

Die reale Belastung der Frauen nach der Geburt von schwerkranken Kindern (unabhängig davon, wie lange sie noch leben) sollte selbstverständlich wahrgenommen und mit entsprechenden Hilfestellungen begegnet werden. Denn die Geburt eines Menschen ist nicht nur die Sache seiner Eltern. Es geht die ganze Menschengemeinschaft an. Staat und Gesellschaft sind immer in die Pflicht dieser Mitverantwortung genommen. Dennoch kann das (vielleicht nur zeitweise) Ausbleiben solcher Unterstützung nicht als Grund für die Abtreibung angesehen werden. Außerdem verwundert es, dass man oft nur von der Belastung der Frau spricht, als ob es grundsätzlich keinen Mann dabei gäbe. Abzuwägen bleibt, ob dies auch nicht mit eine Frucht der moralischen Entwicklung der Gesellschaft ist, in der es immer mehr alleinerziehende Mütter und verantwortungsschwache Väter gibt. Auch hier ist aber die Lösung nicht töten, sondern andere Bedingungen zu schaffen und Familien zu stärken. Samaritanus bonus zeigt mit seiner besonderen Betonung von Integration des ganzen Umfelds des Kindes in den Begleitungsprozess des sterbenden „kleinen Patienten“, wie es möglich ist, die Lasten auf mehrere Schultern zu verteilen und gleichzeitig auf das zu respektierende Ereignis der Krankheit menschlich zu antworten. Eine solche Betonung ist auch ein Zeichen, dass die katholische Kirche nicht bei der starren Verteidigung von abstrakten Prinzipien stehenbleibt, sondern die Nöte der Personen in ihrer Komplexität wahrnimmt und diesen Nöten gemäß dem integralen Wohl der Beteiligten begegnen möchte. 
Vermutlich steckt gerade in der Frage der Relationalität ein bisher wenig ausgeschöpftes Potenzial für die argumentative Kraft des Einsatzes für das Leben der Patienten mit lebensverkürzenden Erkrankungen. In einer verstärkt individualistischen Gesellschaft verliert sich oft die Erkenntnis, dass die der menschlichen Natur zugehörige Sozialität moralische Verpflichtungen auferlegt. Die untrennbare natürliche Bindung der Entstehung von neuen Menschen an den Körper der Frau hat uns entweder etwas Grundlegendes zu sagen, oder muss unbedingt - wie im Rahmen von radikalen emanzipatorischen Bewegungen - überwunden werden. Im zweiten Fall würde man jedoch riskieren, die menschliche Identität grundsätzlich $\mathrm{zu}$ verändern. Will man jedoch diese Identität bewahren, kann man sich im Angesicht des Anderen seiner Verantwortung nicht entziehen, denn als Mensch kann man mit anderen Menschen nicht nicht interagieren. Selbstverständlich bergen solche Interaktionen aufgrund der Unberechenbarkeit des Menschlichen immer ein Risiko in sich. Das Zusammenspiel einer Schwangeren mit ihrem Kind bildet also nur den besonderen Fall dieser tiefhumanen Wahrheit, womit die Rede über moralische Unbedenklichkeit einer freien Verfügung der Frau über ihren Leib im Falle der Schwangerschaft nicht mehr möglich ist.

Das Dokument der Glaubenskongregation stärkt außerdem die Bemühungen, die eine echte Alternative zur Abtreibung darstellen, und zwar die Weiterentwicklung der Palliativmedizin, u. a. mittels der Betreibung von perinatalen Hospizen, welche in Polen seit Jahren mit guten Früchten bestehen. Die Förderung von palliativen Maßnahmen verweist jedoch auch auf die Kehrseite des starken Auftretens für das Lebensrecht von Schwerkranken, eine Kehrseite, die in Samaritanus bonus an mehreren Stellen, auch bei lebensverkürzenden Erkrankungen explizit zum Thema wird. Ebenso gefordert ist nämlich der Verzicht auf jeglichen „therapeutischen Übereifer“, also auf künstliches Verlängern des Lebens eines unabwendbar Sterbenden, womit die Behandlung entweder zwecklos oder mit besonderer Belastung für den Patienten verbunden ist. Die Botschaft ist klar: Wer das menschliche Leben als Fundamentalrecht in allen seinen Ausprägungen schätzt und schützt, wird auch Respekt vor seinem natürlichen Ende zeigen, was ebenfalls zur Achtung der Menschenwürde gehört.

Es verbleibt die größte Herausforderung: der Mentalitätswechsel, auf dessen Bedeutung Samaritanus bonus im Hinblick auf Hindernisse für den Lebensschutz verweist. Der Mensch ist zwar zu vielem fähig, er muss nur darin einen Sinn und einen Wert sehen. Wenn aber die Behinderung eines Ungeborenen als reine Belastung und unerträgliches Schicksal der Frau isoliert dargestellt werden, ohne auf den ontologischen Status des betroffenen Subjekts, geschweige denn Alternativen zu dessen Tötung zu verweisen, dann 
ist es nicht verwunderlich, dass sich die Stimmen, sich des „Problems“ legal zu entledigen, mehren. Obwohl die Denkstrukturen nicht von heute auf morgen verändert werden können, sind jegliche Bemühungen sinnvoll, auf einen solchen Wandel hinzuwirken. Hier öffnet sich ein breites Feld für alle problembewussten Akteure der Gesellschaft, die mit den ihnen gegebenen Mitteln und gemäß der ihnen zukommenden gesellschaftlichen Stellung an solchen Veränderungen mitwirken können.

Zum Schluss ist anzumerken, dass diejenigen, die die dargestellte Argumentation als weltanschaulich nicht neutral betrachten, dem gleichen Vorwurf ausgesetzt sind. Es gibt keine weltanschauliche Neutralität bzgl. der Inhaltsbestimmung von Moral, weil man hier mit der Definition von Gut und Böse konfrontiert ist, welche von anthropologischen und letztlich auch ontologischen Annahmen abhängt: Was ist der Mensch? Was ist sein Ziel? Was ist seine Natur? Ist es sinnvoll, ihn zu schützen? Auf solche Fragen antwortet jeder vor dem Hintergrund bestimmter Voraussetzungen und es besteht kein Grund zu behaupten, dass ausgerechnet die Antworten des katholischen Lehramtes von vornherein ausgeschlossen werden sollten.

\section{LITERATUR}

Bompiani, Adriano. „Indicazioni all'aborto «terapeutico»: stato attuale del problema“. In Aborto. Riflessioni di studiosi cattolici, hrsg. v. Angelo Fiori, Elio Sgreccia, 191-218. Milano: Vita e Pensiero, 1975.

D’Agostino, Francesco. Bioetica. Torino: Giappichelli, 1996.

D'Agostino, Francesco. Parole di bioetica. Torino: Giappichelli, 2004.

Fiori, Angelo und Elio Sgreccia, Hg. Aborto. Riflessioni di studiosi cattolici. Milano: Vita e Pensiero, 1975.

Johannes Paul II. Enzyklika „Evangelium vitae“ über den Wert und die Unantastbarkeit des menschlichen Lebens (25. März 1995). AAS 87(1995): 401-522. Zugriff am 16.12.2020. https://www.vatican.va/content/john-paul-ii/de/encyclicals/documents/hf_jp-ii_enc_25031995 _evangelium-vitae.html.

Kongregation für die Glaubenslehre. „Samaritanus bonus“. Schreiben über die Sorge an Personen in kritischen Phasen und in der Endphase des Lebens (14. Juli 2020). Città del Vaticano: Libreria Editrice Vaticana, 2020. Zugriff am 16.12.2020. https://www.vatican.va/roman curia/congregations/cfaith/documents/rc_con_cfaith_doc_20200714_samaritanus-bonusge. html.

Sgreccia, Elio. Personalist Bioethics. Foundations and Applications. Philadelphia PA: The National Catholic Bioethics Center, 2012. 


\title{
DIE PERSONALISTISCHE GRUNDLAGE DER KIRCHLICHEN LEHRVERKÜNDIGUNG BEZÜGLICH DER PERSONEN MIT LEBENSVERKÜRZENDEN ERKRANKUNGEN
}

\author{
Z u s a m m e f a s s u n
}

Die Protestwelle nach dem Urteil des polnischen Verfassungsgerichts vom 22.10.2020 über die Verfassungswidrigkeit der embryopathischen Abtreibungsindikation kann für die katholische Kirche als Herausforderung betrachtet werden, ihre lebensbejahende Lehre im Hinblick auf den Schutz der Schwächsten zu verdeutlichen und neu zur Sprache zu bringen. Eine Stütze dafür bildet das Schreiben der Glaubenskongregation Samaritanus bonus vom 14.07.2020. Der vorliegende Artikel greift die besagte Lehrverkündigung auf, um die dort vertretene moralische Auffassung bzgl. der Abtreibung, insbesondere im Fall von lebensverkürzenden Krankheiten, zu synthetisieren und zu begründen. Leitend dafür ist die personalistische Sicht des Menschen, nach der die Person im Zentrum aller ethischen Entscheidung stehen soll. Aus der Betrachtung der einzelnen Aspekte der aufgeworfenen Frage ergeben sich bestimmte ethische Imperative, die in den Schlussfolgerungen betrachtet werden.

Schlagworte: lebensverkürzende Erkrankungen; Abtreibung; Person; Bioethik; Relationalität.

\section{PERSONALISTYCZNE PODSTAWY NAUCZANIA KOŚCIOŁA NA TEMAT OSÓB Z WADAMI LETALNYMI}

\footnotetext{
St r e s z c z e n i e
}

Fala protestów po orzeczeniu polskiego Trybunału Konstytucyjnego z 22 października 2020 r. w sprawie niezgodności z konstytucją przesłanki o ciężkim i nieodwracalnym uszkodzeniu płodu jako wskazania do aborcji może być postrzegana jako wyzwanie dla Kościoła katolickiego do sprecyzowania swojego nauczania przyjaznego życiu w odniesieniu do ochrony najsłabszych oraz do podjęcia na nowo tego nauczania. Jedną z pomocy ku temu jest list Kongregacji Nauki Wiary Samaritanus bonus z 14 lipca 2020 r. Niniejszy artykuł odnosi się do wspomnianego nauczania doktrynalnego w celu syntezy i uzasadnienia reprezentowanego w nim stanowiska moralnego na temat aborcji, zwłaszcza w przypadku wad letalnych. Jego zasadą przewodnią jest personalistyczne spojrzenie na człowieka, zgodnie z którym osoba powinna znajdować się $w$ centrum wszystkich decyzji etycznych. $Z$ analizy poszczególnych aspektów postawionego problemu wyłaniają się pewne imperatywy etyczne, które są umieszczone we wnioskach końcowych.

Słowa kluczowe: wady letalne; aborcja; osoba; bioetyka; relacyjność. 